\title{
Full-field in vitro investigation of hard and soft tissue strain in the spine by means of Digital Image Correlation
}

\author{
Maria Luisa Ruspi ${ }^{1}$ \\ Marco Palanca ${ }^{1}$ \\ Cesare Faldini ${ }^{2,3}$ \\ Luca Cristofolini ${ }^{1}$
}

1 Department of Industrial Engineering, School of Engineering and Architecture, Alma Mater Studiorum University of Bologna, Bologna, Italy

2 Department of Biomedical and Neuromotor Sciences, Alma Mater Studiorum University of Bologna, Italy

$32^{\text {nd }}$ Orthopaedic and Traumatologic Clinic, Rizzoli Orthopaedic Institute

Corresponding author:

Luca Cristofolini

Department of Industrial Engineering,

School of Engineering and Architecture

Alma Mater Studiorum - University of Bologna

Viale Risorgimento 2

40136 Bologna, Italy

E-mail: luca.cristofolini@unibo.it

\section{Summary}

Introduction: The spine deserves careful biomechanical investigation, because of the different types of degeneration deriving from daily stress, trauma, and hard and soft tissue pathologies. Many biomechanical studies evaluated the range of motion, structural stiffness of spine segments under different loading conditions, without addressing the strain distribution. Strain gauges have been used to measure strain in the vertebral body, in a pointwise way.

What is currently missing is a method to measure the distribution of strain in the soft tissues (intervertebral discs and ligaments), and an integration between measurements in the hard and soft tissues. Digital Image Correlation (DIC) is a recently developed optical technique, which allows measuring the distribution of displacements and deformation in a contact-less way. It can provide a full-field view of the examined surface under load. DIC can therefore give a more complete knowledge of the biomechanics of the spine.

Methods: This study was performed multisegmental porcine spine specimens with two loading configurations (flexion and lateral bending), while DIC was used to measure the strain distribution. The tests showed the different deformation in the vertebral body, intervertebral discs and ligaments in compression and tension. At the same time it was possible to visualize the growth plates, which are Conclusion: Significantly softer than the vertebral bone.

This work showed the feasibility of investigating the spine in a full-field way, and to quantify the strain inhomogeneity in the vertebrae and soft tissues. Therefore DIC can help improve implantable devices and the surgical technique.

KEY WORDS: biomechanical testing, digital image correlation, intervertebral discs, ligaments, spine, vertebrae.

\section{Introduction}

The spine is one of the most complex structures of the musculoskeletal apparatus with the task of sustaining the body, permitting the movements and protecting the spinal cord. The mechanical structure consists of a sequence of hard tissues (vertebrae) and soft tissue (intervertebral discs), stabilized by other soft tissue (ligaments); they all control the movement in flexion-extension, lateral bending and axial rotation ${ }^{1}$. For instance, failure of posterior fixation and proximal junction kyphosis (PJK) are still unsolved problems ${ }^{2}$. Investigating the biomechanics of the spine is a fundamental task because it could help engineers and clinicians to design implants with a higher success ratio ${ }^{3}$. Spine segments were frequently analyzed in experimental tests applying known motions or known loads ${ }^{4}, 5$. During these tests, stiffness can be evaluated simulating the kinematics of the spine segment as a whole, in physiological conditions, pathological conditions and after treatments conditions ${ }^{6}$.

Strain in the vertebral body can be measured using strain gauges, but these measures are limited to the point of application of the strain gauges 7,8 . A detailed quantification of the local strain distribution could be help to elucidate the failure mechanism and understand the reasons of many post-operative complications. The measurements techniques used so far to measure strains are inadequate in many respects to understand the origin of such problems. Measuring the distribution of strain in the spinal soft tissues (such 
as the intervertebral discs and ligaments) would be a key point. However, this is extremely difficult because of the inhomogeneous and anisotropic properties of such tissues. Strain gauges cannot be used to measure the deformations of the soft tissue, because they would increase the stiffness of the structure and would significantly bias the measurement ${ }^{9}$. Another technique which can be used to measure local strains is Digital Volume Correlation (DVC), which allows measuring strain inside the structure of the vertebral body ${ }^{10}$. Nevertheless, the use of DVC is affected by the time-consuming procedure of images acquisition, which could be a problem with viscoelastic specimens, such as the bone or the intervertebral discs ${ }^{11}$.

Some of unsolved questions about the functionality of the spine concern what happens on the spine segment in terms of local deformation, presence of stress concentration, how the biomechanics of the spine is affected by spinal fixation devices, where the failure point is located (for instance slipped disc, proximal junctional kyphosis, pull-out of the screws, rupture of the bars), or how the spine ligaments work under the different loading conditions. All such clinical problems are not addressed with current in vitro methods $^{2,3,12,13}$. Up to date, an experimental description of the strain distribution on the surface of a spine segment that includes the vertebrae, the discs and the ligaments at the same time is missing. The spine is a combination of different organs (consisting of hard and soft tissues) acting in synergy with a complex biomechanical function. Therefore, it is very important to obtain quantitative and accurate information about the distribution of strain, simultaneously in the hard and soft tissues in order to better understand the behaviour and the failure mechanisms of such a complex structure. A preliminary study has demonstrated that the strain distribution on the surface of an entire spine segment can be measured with sufficient accuracy and precision ${ }^{14}$.

The aim of this work was to explore the feasibility and the potential of using DIC to measure the strain distribution simultaneously on the vertebral bodies, the intervertebral discs, and the spine ligaments of thoracic and lumbar spine segments in different in vitro loading configurations.

\section{Materials and methods}

\section{Specimens}

Three segments of four vertebrae (T7-T10, T11-T14, L2-L5) were extracted from porcine spines. The animals were sacrificed for alimentary purposes. The animals were approximately 9 months old and $100 \mathrm{~kg}$ at sacrifice. The specimens were cleaned using surgical tools: the muscles, the anterior longitudinal ligament, the periosteum and the ribs were carefully removed without damaging the vertebral bodies and the intervertebral discs. Conversely, the interspinous, supraspinous and posterior longitudinal ligaments, and the capsules were left intact in order to preserve the nat- ural kinematics during the tests ${ }^{15}$. The central disc of each segment (respectively, the disc between vertebrae T8 and T9 of segment T7-T10, between T12 and T13 of segment T11-T14, and between L3 and L4 of segment L2-L5) was aligned horizontally in the frontal and lateral views with the use of a six-degree-of-freedom clamp. The extremities of the specimens were potted in poly-methyl-methacrylate (PMMA). The two pots were parallel to one another ${ }^{16}$ (Fig. 1).

\section{Mechanical testing}

Different loading conditions were used to investigate the biomechanics of the multisegmental spine specimens in terms of deformation of the vertebral bodies, intervertebral discs and ligaments ${ }^{17}$. The load was applied using a servo-hydraulic universal testing machine (8032, Instron, High Wycombe, UK) in displacement control. One extremity of the specimen was rigidly fixed to the testing machine while the other extremity was loaded through a spherical joint, which could move on a rail. This system avoided transmission of any other load component.

Two different loading configurations were simulated (Fig. 1) using an eccentric compression load (simulating flexion-extension and lateral-bending), similar to many past studies ${ }^{8}$ :

- anterior bending: the vertical force had an anterior offset equal to the $20 \%$ of the antero-posterior depth of the central intervertebral disc. In this case the rail was set in the anterior-posterior direction;

- lateral bending: the vertical force had a lateral offset equal to the $20 \%$ of the lateral-lateral width of the central intervertebral disc. In this case the rail was set in the lateral-lateral direction.

The load condition did not aim to replicate any specific motor task, but to reproduce simplified scenario and highly reproducible loading conditions, in order to assess the feasibility of using DIC in this kind of application. Once verified the feasibility of DIC measurement, scenarios closer to real motor tasks can be reproduced to obtain useful quantitative information.

Ten preconditioning cycles were applied between 0 and $1.0 \mathrm{~mm}$ of compression at $0.5 \mathrm{~Hz}$. A compression of $3.0 \mathrm{~mm}$ was applied for each loading configuration in $0.1 \mathrm{~mm}$ steps, while DIC images were acquired at each step. The final compression of $3.0 \mathrm{~mm}$ corresponded to a force of the order of $600 \mathrm{~N}$ (approximately $60 \%$ of the animal's body weight) for anterior bending, and of $1100 \mathrm{~N}$ (approximately $110 \%$ of the animal's body weight) for lateral bending. The test was designed to avoid specimen damage, based on some preliminary tests: the strain in the vertebral body did not exceed 2000 microstrain ${ }^{18}$, while strain in the intervertebral discs was below 100000 microstrain (these values were comparable to the values reported in literature associated to physiological load).

\section{Digital Image Correlation}

In order to track the different areas of the specimen surface and compute the displacements and strains, digital image correlation systems require a high-con- 


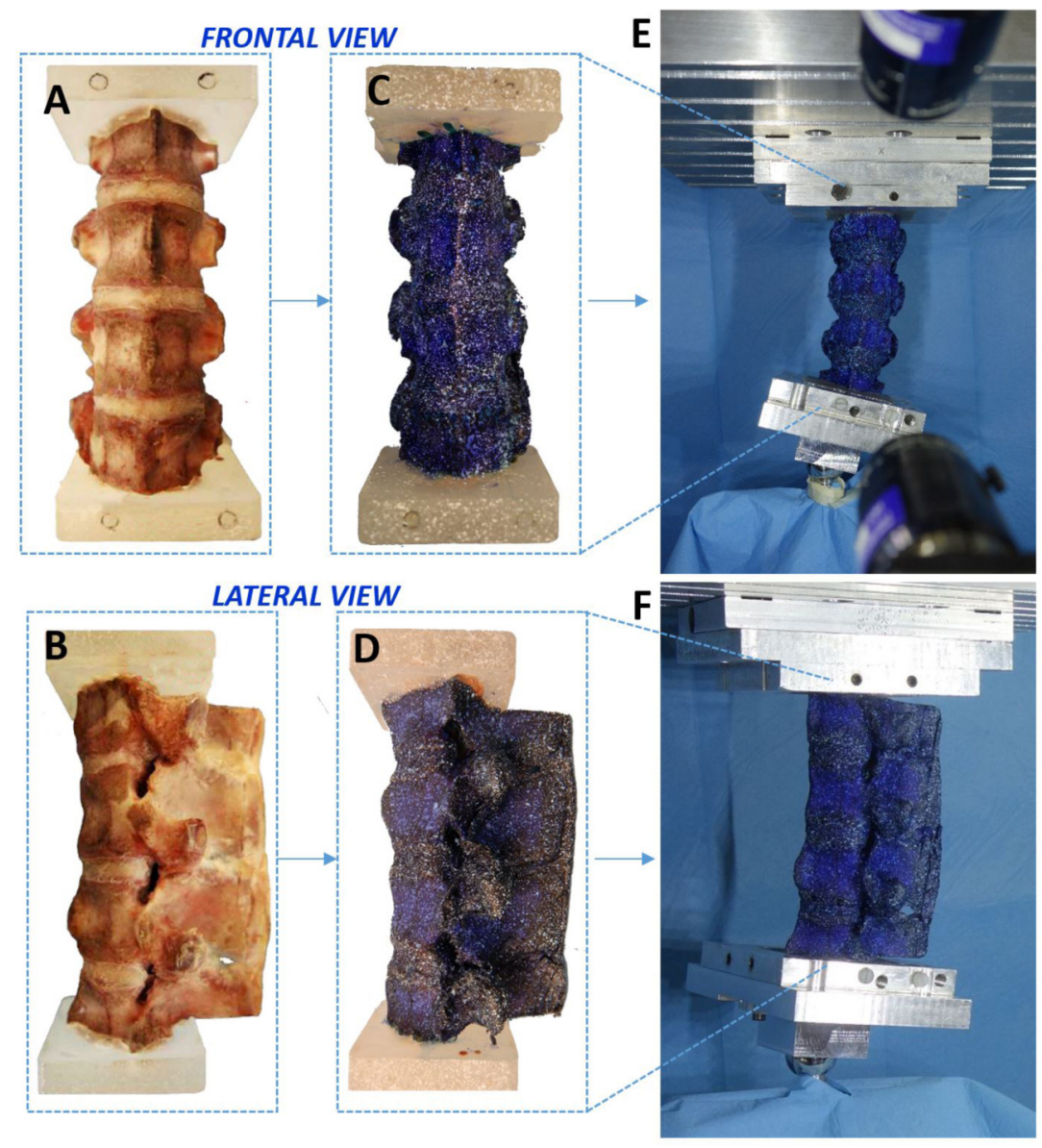

Figure $1 \mathrm{~A}-\mathrm{F}$. The porcine spines were cleaned removing the surrounding tissues $(A, B)$. A white-onblack speckle pattern was prepared, which covered the hard and soft tissues (C, D). Each specimen was subjected to lateral bending $(E$, in a frontal view) and to anterior bending $(F$, in a lateral view). The different loading configurations were reproduced using a universal testing machine and a dedicated system of low-friction linear and ball bearings to avoid transmission of undesired force components.

trast speckle pattern on the region of interest. A white-on-black pattern was prepared on the entire multisegmental spine specimens (both the vertebrae and the intervertebral discs) $)^{10}$ (Fig. 1). The multisegmental spine specimens were first dyed with a dark background, with a solution of methylene-blue and water (4 $\mathrm{g}$ of methylene-blue per $100 \mathrm{ml}$ of water) for three times for the vertebral body and two times for intervertebral discs (to limit as much as possible the increase of stiffness in soft tissues). Methylene-blue preparation has been shown to only marginally alter the mechanical properties of soft tissue ${ }^{19}$.

The white speckle pattern was then applied with the appropriate dot size, following an optimized procedure ${ }^{19,20 .}$.

To measure displacements and deformations, we used a commercial 3D-DIC system (Q400, Dantec Dynamics, Skovlunde, Denmark) with its proprietary software (Instra 4D, v.4.3.1, Dantec Dynamics). To obtain a stereoscopic vision, images were acquired by two cameras (5 Megapixels, 2440 x 2050 pixels, 8-bit) with high-quality $35 \mathrm{~mm}$ lenses (Apo-Xenoplan 1.8/35, Schneider-Kreuznach, Bad-Kreuznach, Germany). The field of view was set to $70 \mathrm{~mm}$ by $60 \mathrm{~mm}$, which gave a pixel size of about 30 micrometers. Calibration was performed before the tests using a dedi- cated calibration target (Al4-BMB-9x9, Dantec Dynamics). To provide sufficient illumination, arrays of cold-light LEDs (10000 lumen in total) were specifically prepared for this test.

The parameters for the acquisition of the images and for the correlation analysis were preliminarily studied and optimized to minimize errors ${ }^{14}$ : facet size: 33 pixels, grid spacing: 19 pixels, contour smoothing: local regression with a kernel size of $5 \times 5$ pixels. These parameters provided a spatial resolution of the order of $3 \mathrm{~mm}$.

The DIC system permitted to investigate the displacement and the strain in a contact-less way providing a full-field view of the examined surface, including the intervertebral discs and the vertebrae (Fig. 1). In order to examine the biomechanical behaviour of the spine, two different acquisitions were performed for each loading configuration and each specimen (Fig. 1):

- frontal view: the cameras pointed to the anterior face of the spine segment;

- lateral view: the cameras pointed to the lateral side (either right or left) of the spine segment.

The tests started from the unloaded condition (reference step, no load applied). A total compression of $3.0 \mathrm{~mm}$ was applied in steps of $0.1 \mathrm{~mm}$. Images were taken at each step with the DIC system. 


\section{Results}

In all the tests, the DIC system permitted to successfully evaluate the deformations of the entire multisegmental spine specimens from a frontal and sagittal view, with different loading configurations. More that $95 \%$ of the region of interest was successfully tracked by the DIC software, providing a truly full-field analysis of the displacements and deformations. Preliminary checks in an unloaded configuration confirmed that the errors with the selected settings did not exceed 140 microstrain.

The three specimens showed similar strain distributions. For all loading configurations, the intervertebral discs and the ligaments reached larger deformations than the vertebral bone of the vertebrae (Figs. 2-5). Since the specimens belonged to young animals, the DIC analysis showed larger deformations of the cartilage part of the vertebrae (growth plate), which are significantly softer than the vertebral bone.

\section{Anterior bending}

During the anterior bending, the intervertebral discs reached larger deformations than the vertebral bone. On the frontal part of the intervertebral discs, the maximum principal strain was in the order of +20000 microstrain (tension) and it was aligned circumferentially, while the minimum principal strain was in the order of -40000 microstrain (compression) and was aligned axially (Fig. 2). On the frontal part of the vertebrae, the strains were one order of magnitude lower: the maximum principal strain was in the order of
+500 microstrain (tension), while the minimum principal strain was in the order of -5000 microstrain (compression) (Fig. 2).

In the lateral sides of the spine segment, the DIC analysis confirmed that there was a strain gradient on the intervertebral discs, with the largest tensile strains in the posterior region, and the largest compressive strain in the anterior region (Fig. 3). The maximum principal strain ranged between $+15000 \mathrm{mi}-$ crostrain (tension) in the median disc of the specimen and +45000 microstrain (tension) in the upper and lower disc of the specimen, while the minimum principal strain was in the order of -50000 microstrain (compression) in the median and lower discs of the specimen. The lateral parts of the vertebrae showed lower strains than the disc due to the grater stiffness of the bone: the maximum principal strain was below +1000 microstrain while the minimum principal strain was in the order of -2500 microstrain (Fig. 3).

In anterior bending, the interspinous and supraspinous ligaments showed large tensile longitudinal deformations: the maximum principal strain was in the order of +45000 microstrain (tension). Additionally, the transversal shrinkage associated with the longitudinal stretching (due to Poisson effect) was visible in the ligaments: the minimum principal strain (compression) was between $-20000 \mathrm{mi}-$ crostrain (in the interspinous ligament) and $-40000 \mathrm{mi}-$ crostrain (in the supraspinous ligament) (Fig. 3).

\section{Lateral bending}

Lateral bending was applied both towards the right and the left sides, to all specimens: the strain distrib-

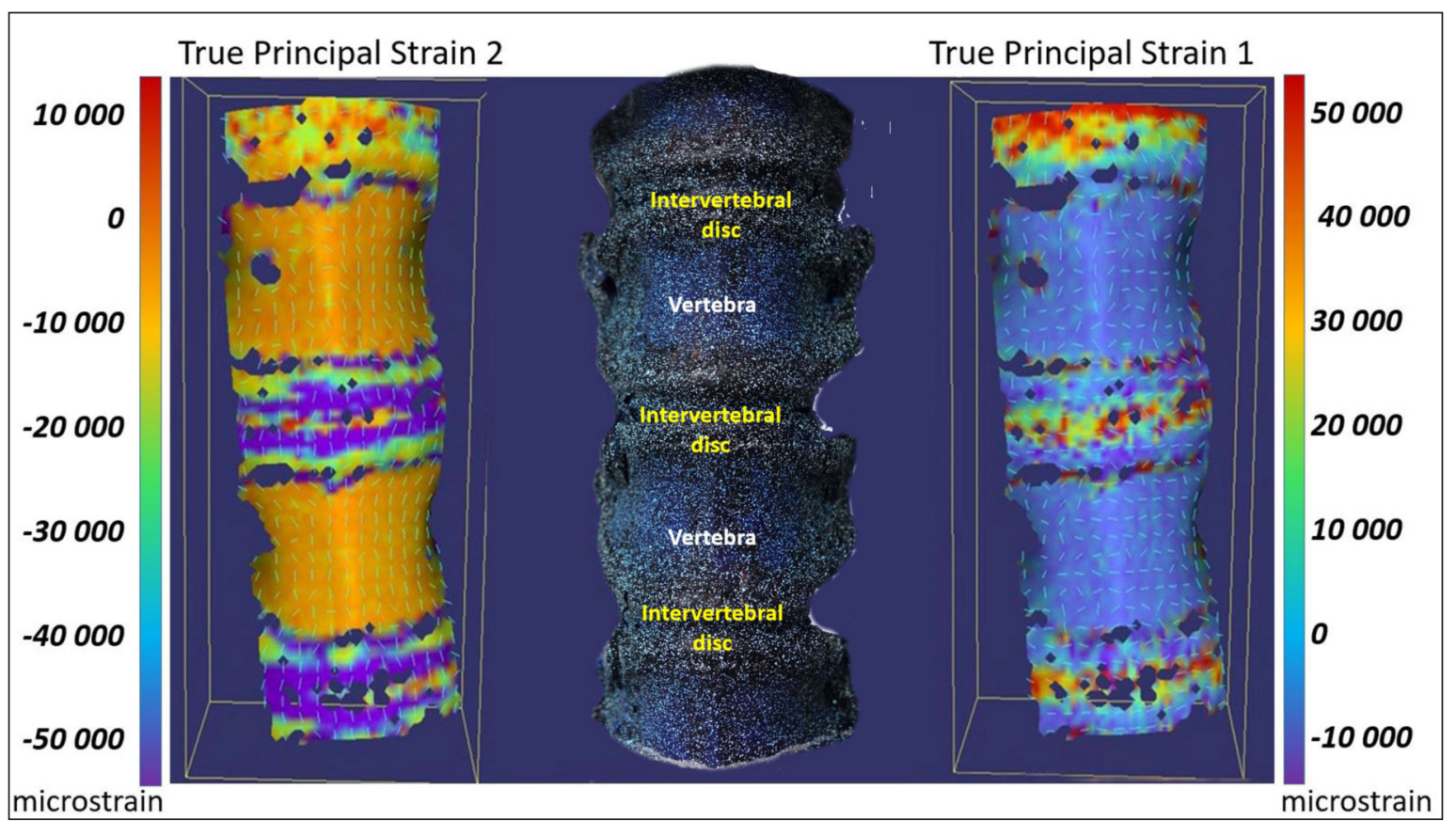

Figure 2. Different deformation of vertebral body, growth cartilages and intervertebral discs during an anterior bending test from a frontal view. The image on the left shows the minimum principal strain (compression), the central image shows the specimen as viewed by the cameras, the image on the right shows the maximum principal strain (tension). 


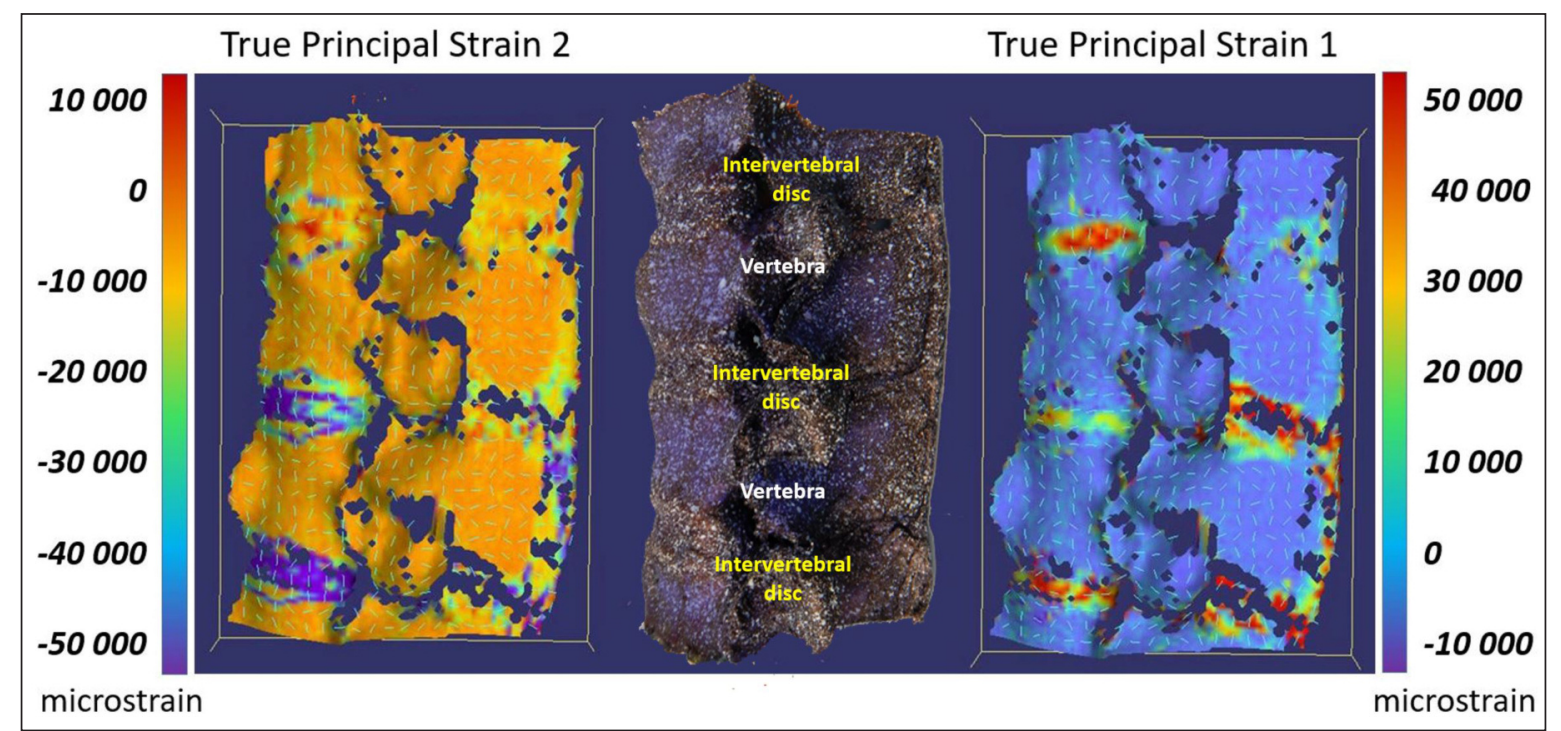

Figure 3. Different deformation of vertebral body, intervertebral discs and ligaments (interspinous and supraspinous ligaments) during an anterior bending test from a lateral view. The image on the left shows the minimum principal strain (compression), the central image shows the specimen as viewed by the cameras, the image on the right shows the maximum principal strain (tension).

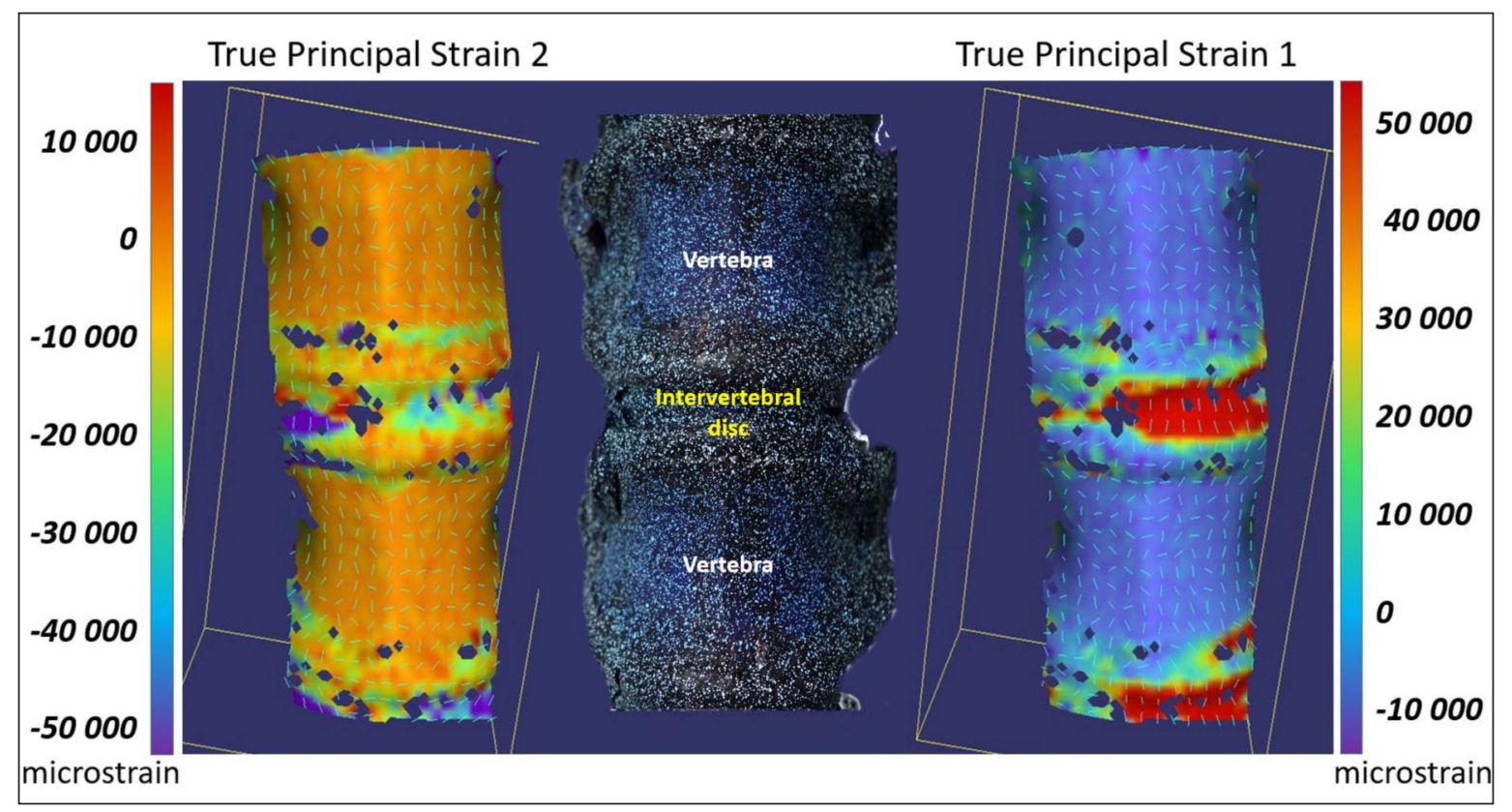

Figure 4. Different magnitudes of strain were visible in the vertebral body, growth cartilages and intervertebral discs during a lateral bending test from a frontal view. The image on the left shows the minimum principal strain (compression), the central image shows the specimen as viewed by the cameras, the image on the right shows the maximum principal strain (tension).

ution measured on intervertebral discs and vertebrae in all the configurations was symmetrical.

In the frontal region, the tensile and compressive strains measured by DIC were consistent with the direction of applied bending (Fig. 4). The maximum principal strain in the discs ranged between +20000 microstrain (tension) in the compressed side to +50000 microstrain (tension) in the stretched side. The minimum principal strains in the discs ranged from -50000 microstrain (compression) in the compressed side to +5000 microstrain (tension) in the stretched side. The orientation of the tensile strain 


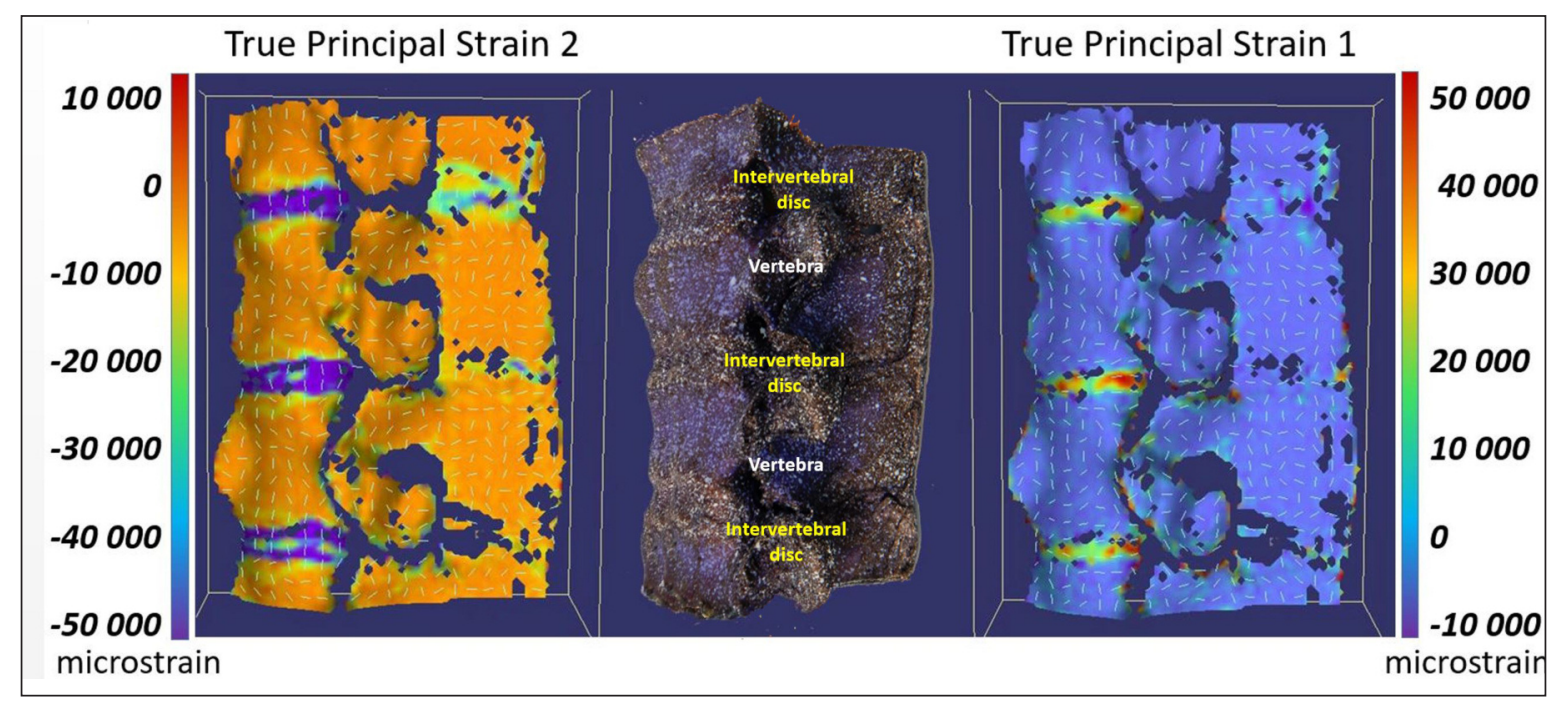

Figure 5. Different magnitudes of strain were visible in the vertebral body, intervertebral discs and ligaments (interspinous and supraspinous ligaments) during a lateral bending test from a lateral view. The image on the left shows the minimum principal strain (compression), the central image shows the specimen as viewed by the cameras, the image on the right shows the maximum principal strain (tension).

changed from circumferential on the compressed side of the disc (due to swelling) to axial on the stretched side (due to traction). The frontal portion of the vertebrae had lower strains than the discs: the maximum principal strains were lower than +500 microstrain (tension) while the minimum principal strains did not exceed -5000 microstrain (compression) (Fig. 4).

In the lateral view, the strain distribution measured on the intervertebral discs was approximately uniform (Fig. 5). The maximum principal strain in the discs reached +45000 microstrain (tension) in the compressed side while the minimum principal strain in the discs was -50000 microstrain (compression) in the compressed side. The lateral parts of the vertebrae had lower strains than the intervertebral discs: the maximum principal strains were lower than $+1000 \mathrm{mi}-$ crostrain (tension) while the minimum principal strains did not exceed -2000 microstrain (compression).

As expected, in lateral bending, the interspinous and supraspinous ligaments showed smaller deformations than in anterior bending: the largest deformation was measured in the interspinous ligament: while the maximum tensile strain (tension) was negligible, the minimum principal strain (compression) was in the order of -25000 microstrain (Fig. 5).

\section{Discussion}

The main aim of this work was to analyze the biomechanics of the spine measuring the strain distribution on thoracic and lumbar multisegmental spine specimens, simultaneously on hard tissues (the vertebral bone) and soft tissues (the intervertebral discs and ligaments) using digital image correlation.

The spines were tested in two different loading con- figurations (anterior and lateral bending), frequently simulated in the biomechanics literature ${ }^{1,6}$. To examine both the hard and soft tissues, the specimens were observed from two different points of view. In all the tests, the DIC system evaluated successfully the deformations of the entire multisegmental spine specimens from a frontal and lateral view. The measurements showed the different magnitude and direction of the strain in the vertebral bones, in the intervertebral discs and in the interspinous and supraspinous ligaments. This confirms the importance of investigating the biomechanics of the spine with a full-field tool. It is worth remarking that the measurement technique adopted is contact-less and causes minimal perturbation to the biological specimen under observation.

The strains measured in the vertebral body were lower than the strains in the intervertebral disc and in the supraspinous and interspinous ligaments. DIC identified that different portions of the intervertebral disc were subjected to compression or tension with different orientations of the principal strain: as expected, in the compressed side of the disc the compressive strains were axial, while circumferential tensile strains were observed. For instance, during the anterior bending, the frontal part of the intervertebral disc was compressed but at mid-height a small region with tensile hoop strain was visible. In fact, when the disc is compressed axially, it swells and the surface expands at mid-height. Since the specimen belonged to young animals, DIC showed also larger deformation in the growth plate than in the neighboring vertebral bone. Also the deformations of interspinous and supraspinous ligaments were successfully measured. As expected, in anterior bending the deformations of these two ligaments were greater than the deformations during lateral bending. At the same time, the transverse shrinkage associated with the longitudinal 
stretching could be observed.

Very few papers can be found in the literature that can be directly compared with our in vitro measurements. In the literature, usually multisegmental spine specimens were investigated in terms of range of motion $^{4,1}$ and stiffness ${ }^{1,21}$. In the vertebrae, strains were evaluated in a point-wise way using strain gauges, which offer an accurate and precise value of the strain, but limited to those points where strain gauges are applied. The ranges of strain measured in the vertebral bone in the present study compare well with the range of strain experience by bone during physiological motor tasks ${ }^{22}$. The values measured in the porcine vertebral bone in the present study are similar to those measured with strain gauges in Cristofolini et al. ${ }^{7}$ even if this study used human lumbar specimens. A problem associated with the use of strain gauges is due to the reinforcement effect caused by their application on the surface. Furthermore, strain gauges cannot be used on the intervertebral discs because the discs have a low elastic modulus and so direct comparison with strains in the intervertebral discs is difficult. Some studies analyzed the vertebral body using DIC system but without taking into account the contiguous intervertebral discs ${ }^{14}$. For example, Gustafson et al. ${ }^{23}$ tested the strains on segments of thoracic and lumbar porcine spine with strain gauges and DIC. While they reported serious problems with their application of strain gauges, direct comparisons are possible with their DIC measurements. The DIC-measured average peak minimum principal strain was -2731 microstrain while the average peak maximum principal strain was 514 microstrain. These values are similar to the values reported in the present study.

The vertebral bone was studied also with the DVC (Digital Volume Correlation). In Danesi et al. ${ }^{24}$ the strain measured in the undamaged region of the vertebral bone of porcine spine did not exceed the value of -2000 microstrain and this result conformed to the values measured in the present study. Another study ${ }^{25}$ performed on human spine, obtained data of strain similar to the ones reported in the present study.

Our measurements concerning the local strain distribution in the intervertebral discs can be compared with few previously published studies: in Spera et al. ${ }^{26}$, they measured the strain distribution in the disc only, whereas their method does not seem applicable to segments comprising both hard and soft tissue. Recently, the feasibility of measuring the full-field strain distribution in the vertebrae and discs by means of DIC was successfully demonstrated ${ }^{27}$.

A limitation of this study is the use of porcine spines instead of human spines. This choice was adopted because this is a preliminary test made to investigate the applicability of the DIC to the vertebral body, the intervertebral disc and the spine ligaments. For ethical motivations, the feasibility study was done on animal specimens, while future studies on the spine biomechanics will be performed on human specimens. Porcine spines are different from human spines in some details (anatomy, different load conditions), but are a valid biomechanical model to demonstrate the feasibility of this new approach ${ }^{28}$. Another limitation relates to the fact that only simplified loading conditions were simulated in this study. However, the proposed approach based on DIC can be used to measure the distribution of strain with any physiological motor tasks (flexion, lateral bending and axial rotation, and any more complex combinations), thus allowing to address the biomechanics of the human spine.

\section{Conclusion}

This study aimed to test the feasibility of using digital image correlation to examine multisegmental spine specimens, with particular attention to soft tissues (intervertebral discs and ligaments). In fact, the strain distribution in the soft tissues cannot be analyzed with the traditional techniques. Surface deformations were successfully measured in the vertebral bones, in the intervertebral discs and in the spine ligaments, highlighting the different magnitude and direction of the strains for the different loading conditions and in the different portions of the spine. The use of DIC can increase the understanding of the biomechanics of the spine, opening the way to new researches in this area (understanding the role of ligaments, studying fixator devices, analyzing failures that occur after surgery), and eventually improving spine treatments.

\section{Ethics}

The Authors declare that this research was conducted following basic ethical aspects and international standards as required by the journal and recently update $\operatorname{in}^{29}$.

\section{References}

1. Busscher I, et al. Biomechanical Characteristics of Different Regions of the Human Spine. SPINE. 2009;34:2858-2864.

2. Kim HJ, et al. Proximal junctional kyphosis as a distinct form of adjacent segment pathology after spinal deformity surgery: a systematic review. Spine (Phila Pa 1976). 2012;37(22 Suppl):S144-164.

3. Lau D, et al. Proximal junctional kyphosis and failure after spinal deformity surgery. SPINE. 2014:39.

4. Panjabi MM, et al. Mechanical Behaviour of the Human Lumbar and Lumbosacral Spine as Shown by Three-Dimensional Load. Displacement Curves. The Journal of Bone and Joint Surgery. 1994:76.

5. Heuer $F$, et al. A new laser scanning technique for imaging intervertebral disc displacement and its application to modeling nucleotomy. Clin Biomech (Bristol, Avon). 2008;23 (3):260-269.

6. Oda I, et al. An In Vitro Human Cadaveric Study Investigating the Biomechanical Properties of the Thoracic Spine. SPINE. 2002:27.

7. Cristofolini $\mathrm{L}$, et al. Strain distribution in the lumbar vertebrae under different loading configurations. Spine J. 2013;13(10): 1281-1292. 
8. Brandolini N, Cristofolini L, Viceconti M. Experimental Methods for the Biomechanical Investigation of the Human Spine: A Review. Journal of Mechanics in Medicine and Biology. 2014;14(01):1430002.

9. Danesi V, et al. Effect of the In Vitro Boundary Conditions on the Surface Strain Experienced by the Vertebral Body in the Eastic Regime. Journal of Biomechanical Engeneering. 2016:138

10. Roberts BC, Perilli E, Reynolds KJ. Application of the digital volume correlation technique for the measurement of displacement and strain fields in bone: a literature review. J Biomech. 2014;47(5):923-934.

11. Palanca M, et al. Digital volume correlation can be used to estimate local strains in natural and augmented vertebrae: An organ-level study. J Biomech. 2016;49(16):3882-3890.

12. Kyaw TA, et al. Biomechanical effects of pedicle screw fixation on adjacent segments. Eur J Orthop Surg Traumatol, 2014;24(Suppl 1):S283-287.

13. Anasetti $F$, et al. Spine stability after implantation of an interspinous device: an in vitro and finite element biomechanical study. J Neurosurg Spine. 2010;13(5):568-575.

14. Palanca M, Brugo TM, Cristofolini L. Use of digital image correlation to investigate the biomechanics of the vertebra. Journal of Mechanics in Medicine and Biology. 2015:15.

15. Behrsin J, Briggs $C$. Ligaments of the lumbar spine: a review. Surgical - Radiologic Anatomy. 1988;10:211-219.

16. Scheer JK, et al. Cervical spine alignment, sagittal deformity, and clinical implications: a review. J Neurosurg Spine. 2013;19(2):141-159.

17. White AA, Panjabi MM. The Basic Kinematics of the Human Spine. SPINE. 1978;3:12-20.

18. Cristofolini L. In vitro evidence of the structural optimization of the human skeletal bones. J Biomech. 2015;48(5):787796.
19. Lionello G, Sirieix C, Baleani M. An effective procedure to create a speckle pattern on biological soft tissue for digital image correlation measurements. J Mech Behav Biomed Mater. 2014;39:1-8.

20. Palanca M, Tozzi G, Cristofolini L. The use of digital image correlation in the biomechanical area: a review. International Biomechanics. 2015;3:1-21.

21. Anderson AL, et al. The Effect of Posterior Thoracic Spine Anatomical Structures on Motion Segment Flexion Stiffness. SPINE. 2009;34:441-446.

22. Lanyon IE. Bone remodelling, mechanical stress, and osteoporosis. Osteoporosis. 1980:129-138.

23. Gustafson H, Siegmund G, Cripton P. Comparison of Strain Rosettes and Digital Image Correlation for Measuring Vertebral Body Strain. J Biomech Eng. 2016;138(5):054501.

24. Danesi V, Tozzi G, Cristofolini L. Application of digital volume correlation to study the efficacy of prophylactic vertebral augmentation. Clin Biomech (Bristol, Avon). 2016;39:14-24.

25. Hussein Al, Barbone PE, Morgan EF. Digital Volume Correlation for Study of the Mechanics of Whole Bones. Procedia IUTAM. 2012;4:116-125.

26. Spera D, Genovese K, Voloshin A. Application of Stereo-Digital Image Correlation to Full-Field 3-D Deformation Measurement of Intervertebral Disc. Strain. 2011;47:e572-e587.

27. Palanca $\mathrm{M}$, et al. Full-field strain distribution in multi-vertebral spine segments: an in vitro application of digital image correlation. Med Eng Phys. 2018;52:76-83.

28. Busscher I, et al. Comparative anatomical dimensions of the complete human and porcine spine. Eur Spine J. 2010;19 (7):1104-1114.

29. Padulo J, Oliva F, Frizziero A, Maffulli N. Muscles, Ligaments and Tendons Journal - Basic principles and recommendations in clinical and field science research: 2016 update. MLTJ. 2016;6(1):1-5. 